\title{
Fast Beam Condition Monitor of the CMS experiment at the HL-LHC
}

\author{
Joanna Wańczyk on behalf of CMS Collaboration ${ }^{a, b, *}$ \\ ${ }^{a}$ École polytechnique fédérale de Lausanne (EPFL), \\ Lausanne, Switzerland \\ ${ }^{a}$ European Organization for Nuclear Research (CERN), \\ Geneva, Switzerland \\ E-mail: jwanczyk@cern.ch, joannawanczyk@epfl.ch
}

To achieve the challenging target of $1 \%$ precision on luminosity determination at the highluminosity LHC (HL-LHC) with an instantaneous luminosity of up to $7.5 \times 10^{34} \mathrm{~cm}^{-2} \mathrm{~s}^{-1}$, the CMS experiment will employ multiple luminometers with orthogonal systematics. A key component of the proposed system is a stand-alone luminometer, the Fast Beam Condition Monitor (FBCM), which is fully independent from the central trigger and data acquisition services and able to operate at all times at $40 \mathrm{MHz}$ providing bunch-by-bunch luminosity measurement. FBCM is foreseen to be placed inside the cold volume of the Tracker as it utilizes silicon-pad sensors exploiting the zero-counting algorithm for luminosity measurement. FBCM will also provide timing information with a few ns precision enabling the measurement of beam induced background (BIB). We report on the optimisation of the design and the expected performance of FBCM.

\footnotetext{
*** The European Physical Society Conference on High Energy Physics (EPS-HEP2021), ***

*** 26-30 July $2021 * * *$

*** Online conference, jointly organized by Universität Hamburg and the research center DESY ***
}

\footnotetext{
${ }^{*}$ Speaker
} 


\section{Introduction}

During Run 2, the instantaneous luminosity at CMS was measured with two dedicated luminometers - Fast Beam Condition Monitor (BCM1F) and Pixel Luminosity Telescope (PLT), and by exploiting measurements of CMS subsystems [1], [2], [3]. The same approach is planned for Run 3. FBCM is planned as a standalone luminometer and BIB monitor for the HL-LHC era [4], as an upgrade of the tried and successful BCM1F. It will not only provide independent operation but also monitoring outside of stable beams and during central DAQ downtimes.

\section{BCM1F}

Based on the Run 2 experience with a mix of silicon and pCVD sensors, BCM1F was re-built for Run 3 as a fully silicon-based system, shown in Fig. 1. Sensors were produced as a part of the Phase 2 Outer Tracker prototype production [5] with the aim to provide a benchmark for the material to be used at the HL-LHC. Double diodes with dimensions of $1.7 \times 1.7 \mathrm{~mm}$ were used, forming a total of 48 independent channels. Sensors have improved radiation tolerance and show excellent signal-to-noise ratio. A 3D printed Ti cooling loop was added, providing active cooling at $-20^{\circ} \mathrm{C}$ with $\mathrm{C}_{6} \mathrm{~F}_{14}$, thus improving longevity. The front end (FE) uses a fast asynchronous ASIC, providing narrow pulses and fast return to baseline. AC-coupling of sensors was added to prevent baseline drift due to leakage current. A $\mu \mathrm{TCA}$ backend system with an improved peak finder algorithm was introduced to enhance the double hit resolution. The system has sub-bunch-crossing time resolution which enables discrimination between the collision products and background, and thus the bunch-by-bunch luminosity and BIB can be measured. The luminosity is obtained with the

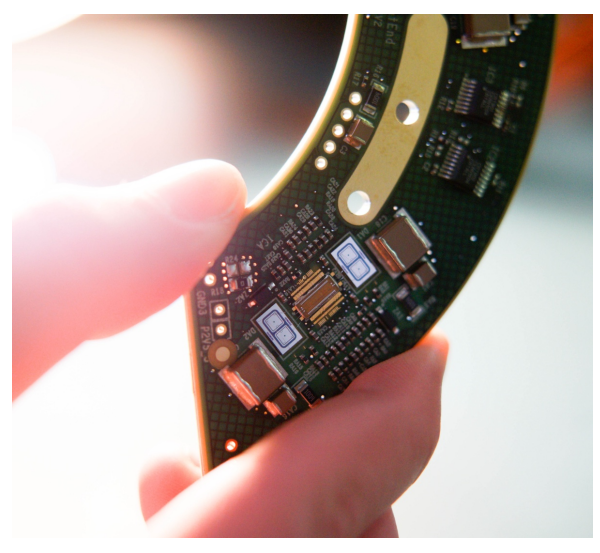

Figure 1: Picture of the Run 3 BCM1F front-end C-shape. One-third of the full module is visible, including the ASIC and two double diodes [6]. "zero-counting" method:

$$
\mu=-\ln [p(0)]=-\ln [1-p(\neq 0)] .
$$

The number of hits per bunch crossing follows the Poisson distribution. Since the occupancy is very low, it is possible to infer the mean value of the number of hits $(\mu)$ by counting the probability of no hits $p(0)$.

\section{Key components of the FBCM design}

The full system is composed of four D-shaped front-end quadrants, shown in Fig. 2. In the baseline design, each quadrant has 4 modules with 21 sensors and 3 FE ASICs (to be customized design for FBCM). Each module is connected to an Inner Tracker port card for opto-transmission, control and monitoring (using IpGBT \& VTRX+ chips) [7]. The full system is made up from a total of 336 silicon-pad sensors. Advanced Telecommunications Computing Architecture (ATCA) blades are planned to be used for signal processing and histogramming the number of hits per BX, 
as presented per quarter in Fig. 3. FBCM is going to be installed very close to the beampipe, at radius of $8.5 \mathrm{~cm}<R<$ $21 \mathrm{~cm}$, and a pseudorapidity of $|\eta| \sim 3.5$, just outside the last Tracker layer, at $z=283.5 \mathrm{~cm}$. The radiation environment was estimated for $4000 \mathrm{fb}^{-1}$, resulting in a total ionizing dose be-
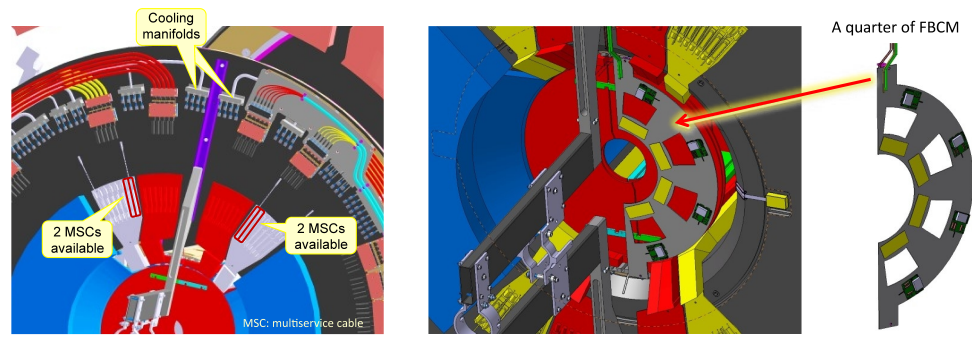

Figure 2: Sketch of a single FBCM quarter at the proposed location with the available infrastructure, behind the last disk of the CMS Inner Tracker Endcap Pixel Detector in the forward region of CMS.

low $200 \mathrm{Mrad}$ and a $1 \mathrm{MeV}$ equivalent neutron fluence below $3.5 \times 10^{15} \mathrm{~cm}^{-2}$.

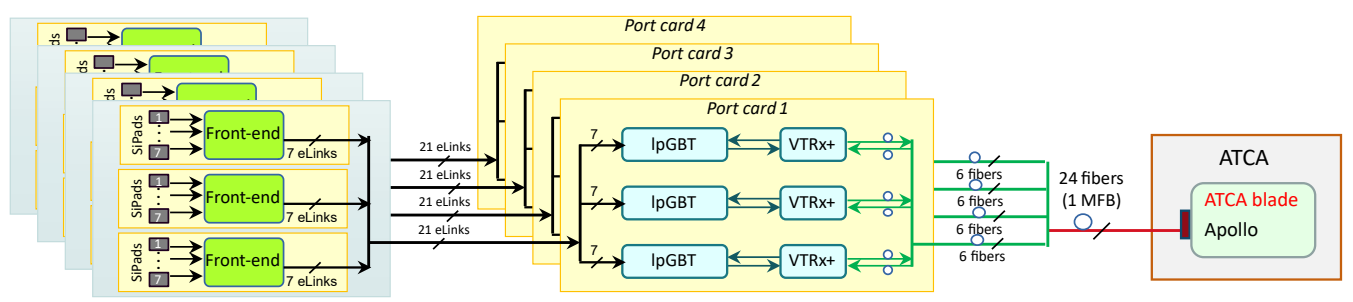

Figure 3: Architecture of a quarter of FBCM. Four portcards, four modules and 84 silicon sensors are used per quarter.

\section{Sensor choice}

AC-coupled sensors with a thickness of $300 \mu \mathrm{m}$ and a size of $2.89 \mathrm{~mm}^{2}$ provide a good compromise between statistical power and linearity over a wide pileup range. Good statistical precision for lowpileup Van der Meer scan (VdM) conditions as well as a low occupancy and unrecognised multiplehit probability were found for a radius of $R=14.5 \mathrm{~cm}$. The simulated metrics used for optimization are shown in Fig. 4, and the preferable placement radius and sensor area are highlighted.

\section{FBCM performance}

The effect of loss of modules as well as aging on luminosity performance were studied. The changes to the rate uncertainty at the maximum pileup and at $\mathrm{VdM}$ conditions as a function of 
the number of failed elements are shown in Fig. 5, showing that FBCM can tolerate the loss of half of its elements. A simulation covering the expected wide range of pileup was performed. Results are shown in Fig. 6 - the mean number of hits per BX per sensor (left), the deviation from linearity (center), and the statistical uncertainty in the rate (right) were estimated. The aging effects on the expected performance of FBCM are also shown for increasing values of integrated luminosity. No significant change to the performance is ex-

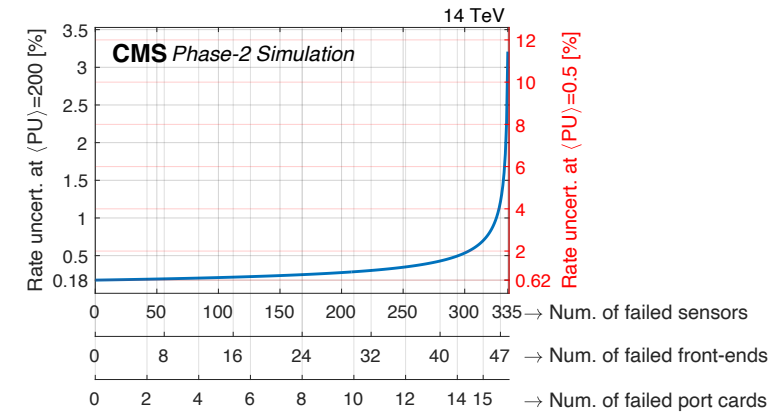

Figure 5: Rate uncertainty per $\mathrm{BX}$ as a function of the number of failed sensors, front-end ASICs, and port cards.

pected. However, degradation of the charge collection efficiency will affect the signal-to-noise ratio.
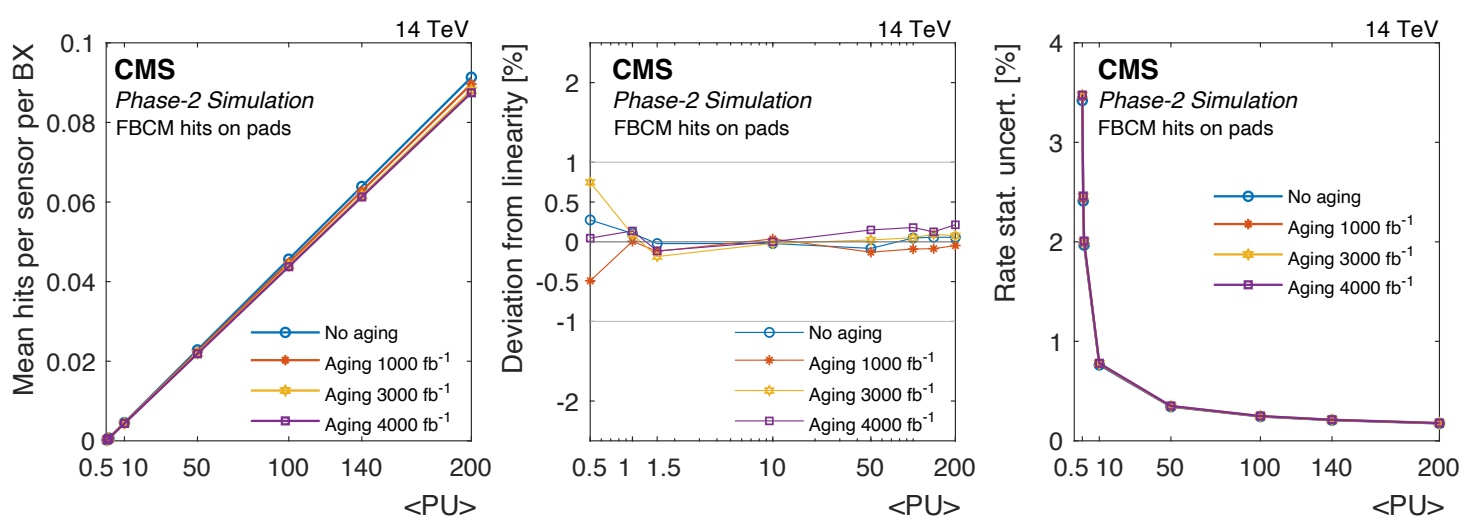

Figure 6: Expected performance of FBCM and the effect of aging for three values of integrated luminosity.

\section{Beam Induced Background}

The BIB is measured either at the beginning of a bunch train or at non-colliding bunches, so that it can be separated from out-of-time contributions (e.g., albedo or afterglow). Two different reference times were considered for the BIB separation. Time of flight (ToF) is defined with respect to nominal collision time at the Interaction Point. The time of arrival (ToA) is the delay with respect to the expected in-time collision products, hence the $\Delta t$ actually measured by FBCM. BIB simulations using CMS reconstruction software (CMSSW) with input from FLUKA [8] were carried out. Using a time binning of $0.78 \mathrm{~ns}$ at $40 \mathrm{MHz}$ rate will allow to identify incoming BIB at a $\mathrm{ToA}=6 \mathrm{~ns}$ with a clear signature.

\section{References}

[1] CMS Collaboration, Precision luminosity measurement in proton-proton collisions at $\sqrt{s}=$ $13 \mathrm{TeV}$ in 2015 and 2016 at CMS. CMS-LUM-17-003. 
[2] CMS Collaboration, CMS luminosity measurement for the 2017 data-taking period at $\sqrt{s}=$ $13 \mathrm{TeV}$. CMS-PAS-LUM-17-004.

[3] CMS Collaboration, CMS luminosity measurement for the 2018 data-taking period at $\sqrt{s}=$ 13 TeV. CMS-PAS-LUM-18-002.

[4] CMS Collaboration, The Phase-2 Upgrade of the CMS Beam Radiation Instrumentation and Luminosity Detectors - Technical Design Report. CERN-LHCC-2021-008, CMS-TDR-023.

[5] CMS Collaboration, The Phase-2 Upgrade of the CMS Tracker. CMS-TDR-014.

[6] BCM1F Picture: CERN Document Server - BRIL LS2 Activity: PLT \& BCMIF Integration

[7] CMS Collaboration, The Phase-2 upgrade of the CMS tracker. CMS-TDR-014.

[8] FLUKA, http://www. fluka.org 